\title{
«Wir waren alleine auf dieser Welt» - Hochzeitsreisen und ihre Settings
}

\author{
Urs Keller, Hans Elsasser, Zürich
}

\section{Einleitung}

Hochzeitsreisen sind ein spezieller, an ein biografisches Fest gebundener Reisetyp. Aus der Sicht des Reisemarktes stellen Hochzeitsreisen ein Nischensegment dar, das weniger durch absolute Zahlen als durch hohe durchschnittliche Ausgaben pro Reise besticht (Blanc 1994; Keller 2007; Liebner 2005). Daten aus den USA (Bulcroft et al. 1999) und den Niederlanden (KaLmiJN 2004) zeigen eine zunehmende Popularität der Hochzeitsreise in den letzten Dekaden. Und auch der Reisemarkt Schweiz weist ein stabiles, bis leicht zunehmendes Marktsegment auf (KelLER 2007). Dieser Befund ist erklärungsbedürftig. Denn die Funktion der Hochzeitsreise lag eigentlich darin, den Neuvermählten einen (Frei-) Raum zu bieten, um sich näher kennenzulernen und sich auf die bevorstehende Haushaltsgründung vorzubereiten (GILLIS 1985; RAPOPORT \& RAPOPORT 1964; SHIELDS 1991; WARD 1990). Mit dem gesellschaftlichen Wandel und der Liberalisierung des Beziehungs- und Sexualverhaltens ging die Hochzeitsreise dieser ihrer Funktion verlustig und wurde, so könnte man meinen, überflüssig. Für die anhaltende Popularität der Hochzeitsreise sind grundsätzlich zwei Erklärungsansätze denkbar. Entweder sie hat sich auf neue Funktionen spezialisiert, weshalb dann von einem Funktionswandel die Rede sein müsste, oder die Hochzeitsreise ist zu einem Klischee verkommen, von dem letztlich nichts als blosser Tourismus übrigblieb (SHIELDS 1991: 151). Neben der grossen Verbreitung der Hochzeitsreise gibt es weitere Gründe, sich mit ihr zu befassen: Für die Tourismuspraxis kann gezeigt werden, dass sich das Geschäft mit den Hochzeitsreisenden lohnt, da mit relativ wenig Aufwand hohe Erträge erzielt werden können (Keller 2007). Für die Tourismustheorie stellt sich die Frage, ob man bei den Hochzeitsreisen überhaupt von einem eigenen Reisetypus mit je eigenen Angebots- und Nachfragemerkmalen sprechen kann. Die Daten muss man dabei selbst erheben, da einschlägige Statistiken und Studien weitgehend fehlen.

Dieser Beitrag geht der Frage nach der Popularität der Hochzeitsreise - und damit auch nach deren Sinn - über die Räume, in denen sie stattfindet, nach. Welches sind die Settings, die für Hochzeitsreisen gewählt werden? Wie sind sie ausgestattet und welche Bedeutung kommt ihnen im Hochzeitsreise-Ablauf zu? Es wird gezeigt, dass die Bühnen und Kulissen für Hoch- zeitsreisen nicht beliebig sind und ihrem «Wesen» nach eine Affinität zum Heiratsritual aufweisen.

Dem Artikel liegt eine breite empirische Basis zu Grunde. (1) Ein Survey unter Heiratswilligen zur bevorstehenden Hochzeitsreise (mit $n=311$ ) lieferte Daten zu den Hochzeitsreise-Destinationen, die im dritten Teil des Artikels näher vorgestellt werden. Diese Destinationen werden in der Analyse zu Settings weiter typisiert. Den Blick auf die Destinationen und deren industrielle Konstruktion schärfte (2) die Marktbefragung. Dazu wurden die wichtigsten Akteure im Schweizer Reisemarkt (mit $n=20$ ) mittels eines schriflichen Fragebogens befragt. Diese Marktbefragung lieferte auch wertvolle Informationen zum Hochzeitsreise-Marketing. Dann wurden (3) narrative Interviews mit 29 Brautpaaren durchgeführt, die nach ihrer Rückkehr in die Schweiz über ihre Flitterwochen zu erzählen bereit waren. Die aufgezeichneten Gespräche wurden transkribiert, wodurch 29 schriftliche Reiseerzählungen vorlagen und der Analyse zugeführt werden konnten. (4) Die Analyse von Bildern in Hochzeitsmagazinen (mit $n=614$ ), gleichsam Repräsentationen von Hochzeitsreisen, rundeten die Datenaufnahme ab. Mit Hilfe dieses triangulativen Forschungsverfahrens sollte eine vertiefte Analyse der Hochzeitsreise-Räume erzielt werden.

Wenn von «Hochzeitsreise» die Rede ist, scheint vorgängig eine Begriffsbestimmung angezeigt. Der Duden definiert die Hochzeitsreise lakonisch als die Reise, «die das Brautpaar [nach der Hochzeit] unternimmt» (Duden 2003: 792). Es ist eine Definition, wie man sie erwarten würde und wie es der Name sagt: die Reise im Zusammenhang mit der anstehenden Heirat. Offen bleibt bei dieser Definition, bis wann genau die Hochzeitsreise abgeschlossen sein muss. In der Praxis werden Hochzeitsreisen nämlich häufig aufgeschoben, für einen Monat, für ein halbes Jahr oder auch länger. Weiter entbehrt die Duden-Definition der Zuschreibung durch die Konsumenten selbst. Auch hier zeigt die Praxis eine kompliziertere Handhabung, als es die nüchterne und einsichtig scheinende Definition vermuten lässt. Es gibt Paare, die eine Reise als Hochzeitsreise bezeichnen, die mehrere Jahre nach der Hochzeit stattfindet, und es gibt Paare, die eine Reise im Anschluss an die Hochzeit unternehmen, diese aber nicht als Hochzeitsreise bezeichnen. Wir halten uns an die alltagsweltliche Praxis und bezeichnen eine Reise dann als Hochzeitsreise, wenn dies die Beteiligten selbst so deklarieren. Der Begriff «Flitterwochen» wird in diesem Artikel synomym dazu verwendet - wie das auch im allgemeinen Sprachgebrauch der Fall ist. 


\section{2 Übergangsrituale und Liminalität}

Die Hochzeitsreise bezeichnet ein Übergangsritual, das den Übertritt in den Verheiratetenstatus begleitet. Der Begriff des Übergangsrituals geht auf den französischen Anthropologen ARNOLd van GenNeP zurück, der ihn mit seinem heute immer noch breit rezipierten Werk «Les rites de passage» zu Beginn des 20. Jahrhunderts eingeführt hat (VAN GENNEP 1986). Übergangsriten begleiten Orts-, Zustands-, Positions- oder Altersgruppenwechsel und vollziehen sich in einem Dreischritt: In der ersten Phase der Trennung löst sich das Subjekt (oder die Gruppe) von einer früheren Position in der Sozialstruktur. In der mittleren Schwellenphase steht das rituelle Subjekt «dazwischen», weder dem früheren noch dem zukünftigen Zustand zugehörig. In der dritten Phase ist der Übergang dann vollzogen; der Ritualteilnehmer oder die Ritualteilnehmerin befindet sich wieder in einem relativ stabilen Zustand mit klar definierten gesellschaftlichen Rechten und Pflichten.

Durch die Vermittlung von Victor Turner fand das van Gennepsche Modell Eingang in die Freizeit- und Tourismusdebatte. Turner (1989) interessierte sich insbesondere für die mittlere Phase, die durch einen Zustand der Liminalität («liminality») geprägt ist. In diesem Schwellenbereich werden die Strukturen des Alltags ausser Kraft gesetzt, indem beispielsweise neue Rollen und Identitäten eingenommen und gewohnte gesellschaftliche Konventionen gesprengt werden. Statussymbole verlieren an Bedeutung, wodurch sich alltägliche, soziale Unterschiede tendenziell einebnen. Liminalität bezeichnet im Kern eine konstruktive Gegenerfahrung zur Normalität - was die Anwendung auf den Tourismus nahelegt (vgl. HenNIG 1997).

Brautpaare befinden sich grundsätzlich in einer liminalen Phase. Die Alltäglichkeit ist mehrfach ausser Kraft gesetzt, was sich etwa in der gängigen Hochzeitsrhetorik zeigt (z.B. «einmal im Leben eine Prinzessin sein»). In der Heiratszeit ist vieles anders: die Zeitstruktur wird verändert, die Publizität des Paars gesteigert - um zwei Beispiele zu nennen. Kaum je wird das Paar gesellschaftlich mehr im Zentrum stehen als zur Zeit des Heiratens. Dem Paar und seiner Liebe wird allenthalben gehuldigt. Mit der Hochzeitsreise wird die Schwellenphase akzentuiert, die Liminalität gesteigert. Das Paar entzieht sich mit der Abreise dem sozialen Umfeld, um sich für eine gewisse Zeit auf sich selbst zu konzentrieren und den besonderen Status in einer anonymen Umgebung zu feiern. Auf der Hochzeitsreise wird mit den letzten Regeln des Alltags gebrochen. Der Aufenthalt in einem luxuriösen Hotel ermöglicht das Eintreten in eine Konsumwelt, die für viele Brautpaare bis dato unzugänglich geblieben ist. Der temporeiche Zeitrhymthus des Hochzeitsfestes wird durchbrochen - sofern man keine geführte Rundreise gebucht hat, die sehr anstrengend sein kann. Wenn der Brautpaarstatus bekannt ist, wird er mit speziellen Annehmlichkeiten sowie mit Lob und Anerkennung seitens des anwesenden Publikums gewürdigt. Mit der Rückkehr in die Schweiz ist das Heiratsritual in der Regel vollzogen. Die Wiederaufnahme des Alltags bedeutet für das neue Ehepaar den Verlust des besonderen Brautpaarstatus. Der Konsumreigen wird gedrosselt, und die gewohnte Hektik hält langsam wieder Einzug. Allerdings ist der Status des Paars ein anderer. Jetzt, im gewohnten Umfeld des Alltags wird sichtbar, was sich formell verändert hat. So wird beispielsweise die (immer noch häufige) Namensänderung der Frau bei der Arbeit auf den Prüfstand gestellt. Das Übergangsritual ist vollzogen.

\section{Hochzeitsreise-Räume}

Im Folgenden wird gezeigt, welche Destinationen Brautpaare für das Übergangsritual «Hochzeitsreise» auswählen. Diese werden sodann zu merkmalstypischen Settings klassifiziert. Weiter wird danach gefragt, wie Paare sich diese Räume in ihrer Hochzeitsreise-Praxis aneignen, welchen Aktivitäten sie nachgehen und wie diese in der retrospektiven Darstellung bewertet werden.

\subsection{Destinationen und Settings}

Die Tabelle 1 zeigt eine Bestandesaufnahme der Reiseregionen, wie sie dem weiter oben vorgestellten Survey entnommen werden kann.

\begin{tabular}{|lc|}
\hline Reiseregion & Anzahl \\
\hline Mittelmeer & 34 \\
Indischer Ozean & 32 \\
Australien/Neuseeland & 18 \\
Nachbarländer (Festland) & 17 \\
Nordamerika & 16 \\
Süd-/Ostasien & 16 \\
Schweiz & 13 \\
Zentralamerika/Karibik & 12 \\
Nordeuropa & 12 \\
Südsee (mit Hawaii) & 11 \\
Südliches Afrika & 6 \\
Diverses & 6 \\
Südamerika & 5 \\
\hline
\end{tabular}

Tab. 1: Reiseziele nach Reiseregionen (Mehrfachnennungen möglich, $\mathrm{n}=178$ )

Destinations de voyages (plusieurs réponses possible, $n=178$ )

Travel destinations (multiple response possible, $n=178$ ) Quelle: eigene Erhebung 
Die Darstellung der populären Flitterwochenziele zeigt einige Auffälligkeiten. $\mathrm{Zu}$ nennen wäre etwa der hohe Anteil an Fernreisen. Ein Vergleich mit den Daten aus dem Reisemarkt Schweiz (BIEgER \& LAESSER 2005) bestätigt diesen Sachverhalt: Im Vergleich zu einem Fernreiseanteil von 10 Prozent bei «normalen» Reisen liegt deren Anteil bei Hochzeitsreisen bei 60 Prozent. Das heisst, das eigene Land ist für Schweizer Brautpaare vergleichsweise eine wenig nachgefragte Reisedestination. Ferner springt die Popularität der «exotischen» Reiseregionen «Indischer Ozean» und «Australien/Neuseeland» ins Auge. Im Grossen und Ganzen kann aber auf der Ebene der FlitterwochenDestinationen eine ansehnliche Variation in der Reisepraxis festgestellt werden.

Anders sieht es aus, wenn man die Destinationen zu Settings typisiert. Dann nämlich zeigt sich die Dominanz zweier Räume, die im Folgenden beschrieben werden.

Den ersten Raum bezeichnen wir als Tropensetting. Es setzt sich aus den Elementen «Meer», «Palmen» und «Strand» zusammen. Dazu gesellt sich ein mehr oder weniger luxuriöses Hotel. Das Farbenspiel, das die Kulisse bestimmt, ist bekannt: Grün (Palmen), Weiss (Sand), Türkis (Meer) und Blau (Himmel) konstituieren ein Bild, welches durch das moderne Reisemarketing unablässig verbreitet wird und welches die Fantasien und Reisesehnsüchte Vieler zu entflammen mag. Der Markt für Hochzeitsreisen konzentriert sich in erster Linie auf Angebote, die einen solchen Raum bereitstellen. Populäre Hochzeitsreise-Destinationen mit Tropensetting sind beispielsweise die Malediven, die Seychellen, Mauritius, die Inseln der Karibik, Bali, Südthailand, Mallorca, Sardinien, Kreta und Santorini. Diese Auflistung an beliebten Hochzeitsreise-Destinationen zeigt zweierlei: 1. Die Angebote decken eine grosse Bandbreite punkto Reisekosten ab. Die meisten Brautpaare, die sich für die Tropen entscheiden, fassen zunächst einmal eine Reisedestination ins Auge, die den «Paradiestraum» zu erfüllen verspricht. Da dies in der Regel exklusive Überseedestinationen in der Karibik, im Indischen Ozean oder in der Südsee sind und diese aus finanziellen Gründen oft ausscheiden, versuchen die Paare, sich mit einem «billigeren» (chartertouristischen) Surrogat dem Traum anzunähern. Das sind dann die Mittelmeer-Inseln, die ebenfalls einen Hauch von «Süden» verströmen. 2. Auffallend oft werden Inseln als Destinationen gewählt. Deren Anziehungskraft wird noch zu klären sein.

Den zweiten, sich einer grossen Nachfrage erfreuenden Raum nennen wir das Wildnissetting. Seine Elemente sind eine weitläufige Landschaft und ihre Zuschreibung als «unberührte» Natur. Der konkrete Landschaftstyp spielt keine grosse Rolle, wenngleich ungewohnte Landschaften wie Wüsten oder Halbwüsten für Mitteleuropäer tendenziell attraktiver wirken als weitläufige Gebirgstopografien oder grössere Waldgebiete. Populäre Flitterwochenziele mit Wildnissetting sind beispielsweise Australien, Nordamerika oder Namibia. Wie beim Tropensetting gibt es auch hier preiswerte Varianten, z.B. Reisen in die Highlands von Schottland oder zu den Seen nach Mittelschweden.

Wiewohl einige Unterschiede zwischen den beiden Settings auszumachen sind - kleinräumige vs. grossräumige Strukturen, geografische Begrenzung vs. geografische Offenheit, Meer vs. Land -, besteht auch eine bedeutende Parallele, die bei der Analyse der Reiseerzählungen sichtbar wird. Diese zeigen nämlich, dass «Natur» bei der Wahl beider Destinationen ein gewichtiges Argument war, und dass Paare bei der Schilderung von Ferienhöhepunkten oftmals «Naturerfahrungen» referieren. Ist diese Natur-Sehnsucht beim Wildnissetting zu einem gewissen Grad nachvollziehbar, ist dies bei den Tropenreisen nur bedingt der Fall. Denn von einer «natürlichen» Umgebung kann schwerlich die Rede sein: Der Strand ist möglicherweise aufgeschüttet, die Palmen fein säuberlich angeordnet und die Hotelanlage mehr oder weniger lieblich in das Ambiente eingebaut. Die Wahrnehmung der Konsumenten wird durch solcherlei anthropogene Einschübe freilich nicht tangiert. Die Hochzeitsreisenden verbinden mit dem Bild des menschenleeren Strandes eine natürliche Umgebung. Genährt wird dieses hochgradig ästhetisierte Bild und dessen Zuweisung als «natürlich» durch massenmediale Erzeugnisse. Alleine mit dem Liebespartner auf einer einsamen, tropischen Insel zu sein - eine ausgesprochen romantische Vorstellung.

Diese Gemeinsamkeit der beiden Settings lässt sich auch umgekehrt herleiten, wodurch die Frage nach der vermeintlichen oder tatsächlichen «Natürlichkeit» obsolet wird. Beide Räume zeichnen sich nämlich durch ihre Gegensätzlichkeit zum städtischen Alltag aus. Sie versprechen den Aufenthalt in einer Umgebung, die sich antithetisch zu den hektischen Grossagglomerationen Westeuropas verhält. In beiden Räumen ist gewissermassen eine Zivilisationsferne eingeschrieben, die sich in erster Linie zwar auf die physische Umgebung bezieht, das soziale Leben aber durchaus miteinschliesst. Auch dieses zeichnet sich durch Marginalität aus; die Einheimischen führen scheinbar ein einfaches, zeitloses Leben - als Landwirte, Fischer oder Hirten. Mit SHIELDS (1991) lassen sich die Hochzeitsreise-Räume daher als «places on the margins» lesen. Diese Orte an der Peripherie weisen einen liminalen Charakter auf, es sind Grenzorte. Einerseits wiederum durch ihre soziale Position im Übergang zur Anökumene, andererseits durch die geografische Lage: das Tropensetting steht am Schwellenraum von Land 
und Wasser, eine Lage die sich im Falle der Insel (bei Hochzeitsreisen wie gesagt ein häufiger Fall) akzentuiert. Hier trennt der Strand das Eiland von den Weiten des Ozeans. Die Bühne der Hochzeitsreise ist weder ganz Land, noch ganz Wasser, ein liminaler Ort par excellence. Aber auch das Wildnissetting bezeichnet einen Schwellenraum. Auch hier bestimmt der Übergang zur zivilisationslosen, «unberührten» Natur die Szenerie. Dazu scheinen die endlosen Weiten Australiens mit dem Horizont zu verschmelzen, was der Landschaft einen erhabenen, quasi-sakralen Zug verleiht. Die Grenzlage beider Hochzeitsreise-Settings zeigt sich zudem in der Paradiesmetapher. Die typischen Hochzeitsreise-Destinationen (oder ihr Marketing) versprechen eine Reise in den Garten Eden bzw. in dessen Vorgärten; weder ganz Paradies (die touristische Infrastruktur mag daran erinnern), noch ganz Erde. Wie die Brautpaare diese temporäre Zeitreise gestalten, zeigt das nächste Kapitel.

\subsection{Die sinnliche Naturerfahrung auf Hochzeitsreisen} In diesem Abschnitt wird aufgezeigt, welche Funktionen Flitterwochen-Grenzräumen zukommen und welche (Reise-)Aktivitäten typischerweise in ihnen stattfinden. Die Ausführungen beruhen auf den Reiseerzählungen, in denen die Paare ihre Ferienerlebnisse und -aktivitäten beschreiben und kommentieren. Die Praxis zeigt, dass Paare vor allem diejenigen Reiseerlebnisse erzählen, die entweder als sehr positiv oder als sehr negativ in das «Feriengedächtnis» eingehen. Damit werden allgemeine Aussagen zu FlitterwochenHighlights möglich. Der Abschnitt zeigt mithin auch, welche Leitthemen Flitterwochen bestimmen. Die Darstellung folgt obigem synthetisierenden Ansatz, beide Settings als Grenz- und Naturräume zu verstehen, und unterscheidet deshalb nicht, ob die Naturerfahrung beispielsweise am Strand oder im Pinienhain gemacht wird. Es wird sich zeigen, dass die darunterliegende Symbolik dieselbe bleibt. Einige Direktzitate der Brautpaare dienen zur Illustration des Gesagten.

Die Natur wirkt in den Flitterwochen einerseits als Kulisse. Die Erlebnisschilderungen zeigen, dass vor dem Hintergrund einer spektakulären Naturkulisse eindrückliche Flitterwochenerfahrungen gemacht werden. Drei Beispiele dazu:

«Bären gingen wir noch anschauen. (...) Zwar war noch nicht richtig Lachssaison und demzufolge noch nicht richtig Bärensaison, aber wir sahen dennoch zwei, drei Bären, nicht gerade die Lachsfischer, weil die Lachse noch nicht so springfreudig waren. (...) Das war fast der lockerste Tag, weil wir etwa fünf Stunden an diesem Wasserfall gestanden sind und einfach gewartet haben» $(1 / \mathrm{m} / 69)$ [Zitatnummer/Geschlecht/Jahrgang].

«Der schönste Moment [war] für mich, als wir in Latsch laufen gingen, in den schönen Wäldern drin. Als wir da einfach auf einer schönen Bank sassen und ins Tal hinab schauten. Und dort hatten wir wirklich eine absolute Ruhe, weil da ist niemand vorbei gekommen. Und dort sassen wir sicher für eine halbe Stunde lang und genossen es. Und die Sonne schien, und das war für mich eigentlich der schönste Moment, halt so richtig nochmals das Verliebtsein auszuspielen» $(2 / \mathrm{w} / 64)$.

Frage: «Was war für dich so der schönste Moment in den Flitterwochen?»

Antwort: «Ja, so das Café, von dem ich bereits erzählt habe. Wo wir viel gefrühstückt haben. Dort zu sitzen, Jazz zu hören und im Hintergrund der Palmenstrand und das Meer. Das war schon sehr schön» (3/w/67). «Sonst, was haben wir noch unternommen? Genossen, viel auswärts gegessen ... wirklich geschaut, dass wir irgendwie ... Brise, Strand, Sonnenuntergang. Die Zeit abgepasst. Weisst, so: Heute kommt der Sonnenuntergang um die Zeit, müssen wir dann und dann ... Und dann wirklich isst du Garnelen und hockst beim Sonnenuntergang ... eben halt die Idylle, so den Klischees nachleben halt» $(3 / \mathrm{m} / 73)$.

Die Paare berichten über Erlebnisse, bei denen die Natur als Kulisse mit Postkartenperspektive wirkt, die betrachtet wird (vgl. URRY 2002). Daneben zeigen die Zitate 2 und 3 die Aktivierung weiterer Sinne. Das Gehör einerseits, wenn sich das Ambiente durch ein «natürliches» Schweigen auszeichnet, und die Gaumenfreuden andererseits, wenn zum Sonnenuntergang Garnelen kredenzt werden. Dass dabei ein Klischee gelebt wird, stört keineswegs.

Weiter wird die Natur als Bewegungs- und Aktionsraum genutzt, wobei auch hier «Natur» sinnlich erfahren und konsumiert wird. Wiederum einige Beispiele:

«Es hatte dort solche Wellenbrecher. Es ist dort die exponierte Seite von Maui, wo es wirklich grosse Wellen hat. Aber durch die Wellenbrecher hattest du vorne so eine Art kleiner Schwimmpool. Und immer wenn die Wellen gekommen sind, sind sie an diesen Felsen gebrochen, und es hat immer ein wenig übergeschwappt. Das war wirklich ein Naturerlebnis, das du auf der Welt nirgends mehr findest. Das war für mich speziell schön. Das warme Wasser, den Wellen zuzuschauen, in diesem Whirlpool zu hocken - das war wirklich super» (5/w/72).

«Dann hat auch die Natur eine wichtige Rolle gespielt. Die Palmen, die rundherum geweht haben, der Sand an den Füssen. Den ganzen Tag einfach barfuss herum zu laufen. (...) Und am Abend wird der Sand kühl, und am Tag ist er warm» $(6 / \mathrm{m} / 72)$.

«Das Zweite ist die Wanderung in dieser Steppenlandschaft auf der Insel draussen. Wir waren da die Einzigen, kein einziger Mensch ist dort gelaufen. (...) Alle waren am 
Meer, und wir sind dort einfach rausgetrampelt, das war sehr lustig.»

Frage: «Was war denn so das Schöne daran?»

Antwort: «Es ist ja topfeben das Land, und du siehst so weit und hast keinen einzigen Menschen gesehen. Ich habe gedacht, wir sind jetzt ganz alleine auf dieser Welt» $(7 / \mathrm{w} / 75)$.

Die Aktivitäten in der Natur sind typischerweise Spaziergänge in der «wilden» Natur oder am Strand auf der einen Seite und Erlebnisse am und im Wasser auf der anderen Seite. Zu Letzteren gehört z.B. das Schnorcheln, Tauchen und Baden. Die Natur wird auch bei den hier skizzierten Erlebnissen sinnlich wahrgenommen: Das warme Wasser im «natürlichen» Whirlpool, die warme Tropenluft, der warme bzw. kalte Sand an den Füssen. Das Zitat 7 zeigt die Verknüpfung zweier Leitbilder der modernen Hochzeitsreise: die sinnliche Naturerfahrung und die Erfahrung von Zweisamkeit. Die Erfahrung, alleine mit dem Liebespartner in der weiten Natur zu sein (hier: in einer Steppenlandschaft in Skandinavien), wird in vielen Reiseerzählungen unter die Highlights eingereiht. Die Schilderungen wecken wiederum Reminiszenzen an den Aufenthalt des ersten Menschenpaars im Garten Eden; für einen Augenblick wird das verlorene Paradies zurückerobert. Dies sind Schlüsselmomente auf dem Weg zu einer erfolgreichen Hochzeitsreise.

Mitunter stellt sich ein Zustand ein, bei dem das Innenleben der Reisenden mit der atmosphärischen Stimmung zu verschmelzen scheint und das Gefühl evoziert wird, in Einklang mit der Natur zu sein:

«Die Trauung war ein Wahnsinnserlebnis. Einfach die Stimmung und das Gefühl, das man hat, das Gefühl war super intensiv. Ein bewegender Moment im Leben irgendwo auch, halt auch ein Schritt trotz allem. Und die Landschaft an sich, es hat so gestimmt. Das Gefühl, das ich in mir drin gehabt habe und in dieser Umgebung, wo ich gewesen bin. (...) Es war superschön» (8/w/72).

Sowohl vonseiten der wissenschaftlichen Theorie als auch vonseiten der Beteiligten lassen sich die hier skizzierten und illustrierten Erlebnisse als romantischen Erfahrungskonsum einordnen. Naturerfahrung und Zweisamkeit - oft verbunden mit den Lockungen des Warenkonsums (Garnelen) - sind Leitbilder des westlich kodifizierten Liebesrituals (Illouz 2003). Ohne den Sachverhalt näher erläutern zu können, befinden die Brautpaare diese Momente des Glücks für romantisch, selbst dann, wenn sie einsehen, dass diese Einschätzung weniger eine individuelle als eine gesellschaftliche ist (s. Zitat 4). Als Paradebeispiel mag der Strandspaziergang gelten. Das Bild des Paars, das bei Sonnenuntergang einsam den Strand entlang schlendert, ist fest in unseren Köpfen verankert, vermittelt durch den Reisemarkt und die Kulturindustrien (allen voran die Massenmedien). Zuweilen hängt das Bild bereits im Teenager-Zimmer und vermag so Fantasien und Geschichten auszulösen, die in den Flitterwochen einzulösen versucht werden:

«Die Bilder vom Strand mit türkisblauem Wasser, weissem Strand, Palmen und die einsame Insel. Das war schon etwas, wo ich gedacht habe: Doch, spätestens bei der Hochzeitsreise könnte ich es mir vorstellen, an einen solchen Ort zu gehen. Ich habe lange zu Hause Bilder von den Virgin Islands aufgehängt gehabt, wunderschön, und gedacht, das sei mal eine Destination für eine Hochzeitsreise. Das hatte ich mir immer so romantisch ausgemalt» $(9 / \mathrm{w} / 72)$.

\section{Fazit}

Die Hochzeitsreise ist ein Schwellenritual, das einen geordneten Übergang in den Verheiratetenstatus sicherstellt. In mannigfacher Weise hilft die Hochzeitsreise mit, den biografischen Schritt zu erleichtern. Sie bietet eine Plattform, die schnell ablaufenden Ereignisse rund um die Hochzeit nochmals Revue passieren zu lassen und nach Aussagen der Betroffenen zu «verarbeiten»; sie bietet eine Plattform, um sich als neuvermähltes Paar einer unbekannten Öffentlichkeit zu präsentieren, den neuen Status auszutesten («mein Mann», «meine Frau») und damit Paaridentität aufzubauen; und sie bietet eine Plattform, um den Ehestart zu einem besonderen, romantischen Ereignis zu machen. Am Anfang der Ehe steht ein Liebesritual, das die Ehe in gute Bahnen lenken soll. Einigen Brautpaaren war schon kurz nach der Hochzeitsreise klar: Dieses einmalige Erlebnis wird in die Paargeschichte eingehen und einen Ankerpunkt bieten, an den man sich in stürmischeren Ehezeiten halten und sich der unsterblichen Liebe zu Beginn der Ehe entsinnen kann.

Für dieses Schwellenritual wählen die Brautpaare ihr Setting mit Bedacht. Die teils erheblichen zeitlichen Investitionen in die Wahl der Reisedestination zeugen davon. Die diesem Artikel zugrunde liegenden Daten zeigen eine starke Normierung bezüglich der Settings in Richtung einer quasi-natürlichen Umgebung, die sich vor allem durch den Antagonismus zur städtischen Lebenswelt auszeichnet. Hierin liegt ein bedeutender Unterschied zum «gewöhnlichen» Reiseverhalten, wie es im Reisemarkt Schweiz zum Ausdruck kommt. Die nachgefragten Räume und die damit verknüpften Aktivitäten sind weitaus vielfältiger als dies bei Hochzeitsreisen der Fall ist. Klassische Reisetypen und -settings wie z.B. Städtereisen, Einkaufs- und Eventtrips oder der Aufenthalt in Themenparks sind bei Hochzeitsreisen selten (vgl. BIEGER \& LAESSER 2005: 23). 
Die zwei dominanten «Natur»-Settings der Hochzeitsreisen lassen sich zu Grenzräumen weiter verdichten. Es sind solcherlei Zwischenorte, die als FlitterwochenBühne fungieren; für das Schwellenritual Hochzeitsreise werden Schwellenräume gewählt. Die moderne Hochzeitsreise zeichnet sich überhaupt durch ihren Kontrast zum Alltag aus. Der besondere Brautpaarstatus - durch den Mythos der Einmaligkeit alimentiert - erfordert ein spezielles Setting, in dem ausserordentliche Erlebnisse stattfinden können. Die romantische Utopie (Illouz 2003) und ihr kulturell kodifizierter Formenschatz stellen die entsprechenden Drehbücher bereit: man braucht nur noch auszuwählen. Die Flitterwochen-Kulissen und die einschlägigen Verhaltensweisen in Richtung des romantischen Erfahrungskonsums findet man in Hochzeitsmagazinen, Fernsehsendungen und Reisekatalogen. Nach der Hochzeitsreise ist der Spuk vorbei. Mit dem Verlassen des FlitterwochenGrenzortes und der Ankunft zu Hause geht die Schwellenphase des Heiratsrituals zu Ende. Die Reintegration in den alten, neuen (Ehe-)Alltag beginnt.

\section{Literatur}

Bieger, T. \& C. Laesser (2005): Travel market Switzerland. Basic report and database specification. - St. Gallen: Institut für Öffentliche Dienstleistungen und Tourismus (IDT).

Blanc, E. (1994): Un marketing spécifique pour un marché prometteur. - In: Espaces 126: 18-19.

Bulcroft, K., Smeins, L. \& R. Bulcroft (1999): Romancing the honeymoon. Consummating marriage in modern society. - Thousand Oaks, London, New Delhi: Sage.

Duden (2003): Das grosse Wörterbuch der deutschen Sprache. - Mannheim, Leipzig, Wien, Zürich: Dudenverlag.

GILLIS, J.R. (1985): For better, for worse. British marriages, 1600 to the present. - New York: Oxford University Press.

Hennig, C. (1997): Reiselust. Touristen, Tourismus und Urlaubskultur. - Frankfurt am Main, Leipzig: Suhrkamp.

Illouz, E. (2003): Der Konsum der Romantik. Liebe und die kulturellen Widersprüche des Kapitalismus. - Frankfurt am Main, New York: Campus.

KaLmiJn, M. (2004): Marriage rituals as reinforcers of role transitions: an analysis of weddings in the Netherlands. - In: Journal of marriage and the family 66: 582594.

Keller, U. (2007): «Wenn die Liebe auf Reisen geht»Zur Praxis der Hochzeitsreisen von Schweizer Brautpaaren. - Zürich: Offizin.

Liebner, B. (2005): Der Markt für Hochzeitsreisen eine tourismuswirtschaftliche Betrachtung. - Unveröffentlichte Diplomarbeit am Lehrstuhl für Tourismuswirtschaft der Technischen Universität Dresden.
RAPOPORT, R. \& R.N. RAPOPORT (1964): New light on the honeymoon. - In: Human relations 17, 1: 33-56.

SHIELDS, R. (1991): Places on the margin. Alternative geographies of modernity. - London, New York: Routledge.

Turner, V. (1989): Das Ritual. Struktur und AntiStruktur. - Frankfurt am Main, New York: Campus. URRY, J. (2002): The tourist gaze. - London: Sage. VAN GenneP, A. (1986) (frz. 1909): Übergangsriten (Les rites de passage). - Frankfurt am Main: Campus. WARD, P. (1990): Courtship, love, and marriage in nineteenth-century English Canada. - Montreal: McGillQueen's University Press.

\section{Zusammenfassung: «Wir waren alleine auf dieser Welt» - Hochzeitsreisen und ihre Settings}

Hochzeitsreisen sind ein spezieller, an ein biografisches Fest geknüpfter Reisetyp. Kulturanthropologisch gesehen bezeichnen Hochzeitsreisen ein Übergangsritual. Vor dem Hintergrund des gesellschaftlichen Wandels und einem liberalisierten Beziehungs- und Sexualverhalten stellt sich die Frage, weshalb sich Hochzeitsreisen einer steigenden Beliebtheit erfreuen; verschiedene Studien zeigen, dass die Nachfrage nach Hochzeitsreisen in den letzten Jahren zugenommen hat. In diesem Beitrag wird der Blickwinkel auf die Räume gerichtet, in denen Hochzeitsreisen stattfinden. Die Analyse der Destinationen und Settings soll einen Beitrag zur Frage nach der Popularität der Hochzeitsreisen leisten. Auf der Grundlage von narrativen Interviews, Surveydaten und einer Marktbefragung wird gezeigt, welches beliebte Flitterwochenziele sind und welche Räume dafür gewählt werden. Darauf aufbauend wird der Frage nachgegangen, welche Funktionen die beschriebenen Räume im Hochzeitsreise-Ablauf innehaben, und welche Reiseaktivitäten in ihnen ausgeübt werden. Der Artikel typisiert die dominanten Settings der Hochzeitsreisen zu Grenzräumen, die sich durch Zivilsationsferne auszeichnen. Ihre Anziehungskraft beziehen sie aus der Antithese zur gewohnten grossstädtischen Lebenswelt. Diese «natürlichen» Umgebungen stellen die Bühne und Kulisse für ein zentrales Postulat der Hochzeitsreise bereit: den Neuvermählten ein romantisches Erlebnis $\mathrm{zu}$ ermöglichen und so die junge Ehe in gute Bahnen zu lenken.

\section{Résumé: «Nous étions seuls au monde». Les voyages de noces et leurs préparatifs}

Les voyages de noces forment un type de voyage tout à fait spécial, qui participe de l'histoire intime des conjoints. Les anthropologues considèrent d'ailleurs ces voyages comme des rites de passage. Dans un contexte de changement social et une libéralisation des relations sexuelles et des comportements, la question se pose de savoir pourquoi les voyages de noces 
jouissent d'une popularité croissante, cette dernière ayant été relevée par plusieurs études au cours de ces dernières années. Dans ce contexte, cette contribution étudie les différents espaces dans lesquels se déroulent les voyages de noces. L'analyse des destinations et des préparatifs permet d'apporter une contribution à la question de savoir pourquoi les voyages de noces sont toujours aussi populaires. Sur la base d'entretiens, d'enquêtes statistiques et d'une étude du marché, l'article détaille quelles sont les destinations favorites des lunes de miel et quels espaces sont préférentiellement choisis par les jeunes mariés. C'est alors l'occasion de montrer quelles sont les caractéristiques des lieux sélectionnés dans le voyage, de même que les activités qui s'y déroulent. L'article montre en particulier quels sont les types de préparatifs récurrents dans les voyages de noces. Les espaces privilégiés pour ce genre d'activités se situent à l'antithèse des grands espaces urbanisés. Le rôle de ces espaces «naturels» est de mettre en scène le postulat central du voyage de noce: permettre une aventure romantique aux jeunes mariés et conduire ainsi le jeune couple sur la bonne voie.

\section{Abstract: «We were alone here on Earth» - Honey- moon trips and settings}

Honeymoons are special, biographically-fixed forms of travel. From the viewpoint of cultural anthropology, a honeymoon may be seen as a transitional rite. Taking cultural change and the liberalised relational and sexual behaviour of today into consideration, it is interesting to note that honeymoons are increasingly popular. Several studies have drawn attention to the increased demand for honeymoon trips. This article focuses on the space in which honeymoons take place. The analysis of destinations and settings can offer an explanation for this increasing popularity. This article makes use of qualitative interviews, survey data and a market survey to assess popular destinations and spaces. Further, the function taken on by the chosen spaces and their role within the honeymoon phase are explored. As a consequence, popular honeymoon destinations are defined as border areas characterised by «distance from civilisation». Their attractivity draws on their starkness of contrast to every-day urban life. These «natural» areas set the stage for a central role of honeymoons - to allow newlyweds a romantic experience that will set the foundations for their young marriage.

Dr. Urs Keller, Prof. Dr. Hans Elsasser, Geographisches Institut der Universität Zürich, Abteilung Wirtschaftsgeographie, Winterthurerstrasse 190, CH-8057 Zürich, Schweiz.

e-mail:

urs.keller@geo.uzh.ch

hans.elsasser@geo.uzh.ch

\section{Manuskripteingang/received/manuscrit entré le} 29.5.2007

Annahme zum Druck/accepted for publication/accepté pour l'impression: 3.12.2007 